\title{
Shave Biopsy
}

National Cancer Institute

\section{Source}

National Cancer Institute. Shave Biopsy. NCI Thesaurus. Code C15387.

Removal skin tissue, for microscopic examination, using a blade oriented in the horizontal plane of the skin surface. 